\title{
Représentations de l'espace dans les sciences humaines
}

\section{Alberto Roncaccia}

\section{(2) OpenEdition}

\section{Journals}

Édition électronique

URL : http://journals.openedition.org/edl/476

DOI : $10.4000 /$ edl. 476

ISSN : 2296-5084

\section{Éditeur}

Université de Lausanne

\section{Édition imprimée}

Date de publication : 15 mai 2013

Pagination : 11-16

ISBN : 978-2-940331-32-1

ISSN : 0014-2026

Référence électronique

Alberto Roncaccia, «Représentations de l'espace dans les sciences humaines », Études de lettres [En ligne], 1-2 | 2013, mis en ligne le 15 mai 2016, consulté le 19 décembre 2020. URL : http:// journals.openedition.org/edl/476 ; DOI : https://doi.org/10.4000/edl.476 


\title{
REPRÉSENTATIONS DE L'ESPACE DANS LES SCIENCES HUMAINES
}

\begin{abstract}
Principio, dove io debbo dipingere scrivo uno quadrangolo di retti angoli quanto grande io voglio, el quale reputo essere una finestra aperta per donde io miri quello che quivi sarà dipinto. Leon Battista Alberti, De pictura ${ }^{1}$.
\end{abstract}

Organisé en 2011 par la Formation doctorale interdisciplinaire de la Faculté des lettres (UNIL), le colloque «La représentation de l'espace dans les sciences humaines" souhaitait interroger et mettre en relation l'image et la notion d'espace transmises par les discours spécifiques aux disciplines littéraires, linguistiques et historiques. Les participants ont été invités à étudier des situations où le moi (observateur ou observé, individuel ou collectif) se définissait en fonction de dispositifs d'orientation et d'identification, en accord ou en désaccord avec son groupe d'appartenance. La réflexion mettait aussi l'accent sur le fait que tout discours est tributaire des indicateurs de direction que la langue et la culture mettent à sa disposition.

Les actes de ce colloque sont réunis sous le titre de «Représentations de l'espace». Un deuxième volet, intitulé "Visions du paysage», comprend un choix parmi les interventions qui ont eu lieu, toujours en 2011, lors d'une autre initiative interdisciplinaire, le «Laboratorio

I. "Mon premier acte, quand je veux peindre une superficie, est de tracer un rectangle, de la grandeur qui me convient, en guise de fenêtre ouverte par où je puisse voir le sujet" (Leon-Battista Alberti, De la statue et De la peinture, traités trad. du latin en français par Claudius Popelin, Paris, A. Lévy Editeur, 1869, p. 124. Le texte original est tiré de Leon Battista Alberti, De pictura, a cura di Cecil Grayson, Bari, Laterza, 1975, p. 36). 
internazionale sul paesaggio", organisée à San Biagio della Cima par plusieurs institutions académiques et culturelles.

L'ensemble des essais fournit un échantillon de l'insaisissable multiplicité des «visions» de l'espace et du paysage. Pour différencier ces deux notions, nous pouvons néanmoins nous référer à un passage du sémiologue Jacques Fontanille qui ouvre la deuxième partie de la présente publication:

Un paysage se présente d'abord comme un segment du monde naturel, un espace figuratif, dont la valeur émane des sensations et perceptions qu'il procure, eu égard à une position d'observation ${ }^{2}$.

$\mathrm{Si}$, pour un instant, nous attribuons au passage cité un sens légèrement axiomatique, ces deux notions semblent se disposer naturellement selon une partition logique. Le paysage est, comme l'explique Fontanille, un "espace figuratif", c'est-à-dire un espace encadré par un point d'observation précis et procurant à l'observateur un plaisir (ou éventuellement un déplaisir) cohérent. Et il est utile de préciser qu'un "espace produit» de type urbain, industriel, agricole, peut également être perçu comme un segment du monde naturel que le sujet reconnaît grâce à un ensemble cohérent de sensations et de perceptions.

En procédant par soustraction, nous pouvons remarquer que la notion d'«espace» n'implique pas nécessairement, de la part de l'observateur, l'attribution d'une valeur à la qualité de l'expérience émotionnelle procurée par la perception d'une partie délimitable du monde. Face au monde, l'observateur continue cependant d'effectuer un acte de cadrage. Pour cette raison une simple idée d'extension, limitée ou illimitée, reste inadéquate à expliquer la notion d'espace.

La notion d'espace qui semble le mieux convenir à l'ensemble des articles proposés est celle d'un système d'usages ou de forces qui règlent un groupe d'événements pendant une certaine durée. Comprendre un espace donné signifie donc prendre conscience des mouvements et des jeux de forces (physiques, politiques, économiques, linguistiques, culturelles, etc.) qui établissent, pour un temps déterminé, un système d'interactions possibles.

2. Cf. infra J. Fontanille, «Paysages: le ciel, la terre et l'eau», p. 231. 
C'est selon cette hypothèse de travail que la partition entre "visions de l'espace» et «visions du paysage» peut trouver son sens.

Le volet "Représentations de l'espace» est introduit par Bertrand Westphal qui propose une réflexion sur l'artificialité de tout processus de «territorialisation» implicite dans la représentation cartographique. Ce processus est déconstruit dans ses fondements idéologiques, voire "coloniaux", à travers son application artistique à des objets inappropriés, comme par exemple le corps humain.

Dans l'article suivant, Maria Grazia Bianchi analyse quelques descriptions géographiques inédites du XVI siècle qui traitent de l'Europe de l'Est, en particulier de la Hongrie, de la Transylvanie, de la Pologne et de la Bohème. Ces documents montrent une vision géostratégique cohérente de l'espace européen, basée sur des critères d'observation qui doivent permettre aux destinataires occidentaux de mesurer la solidité politique et économique de régions dont on avait, jusqu'à cette époque, une connaissance approximative. Ces espaces politiques entrent ainsi dans une relation de continuité vis-à-vis du centre politique, culturel et religieux de l'Europe occidentale à l'époque du concile de Trente.

Dans la troisième étude, Marisa Gazzotti montre la progressive hiérarchisation de l'espace de la cour parisienne d'Henri III, en utilisant comme source les rapports inédits d'un diplomate italien au service des seigneurs de la Mirandole, petit Etat italien satellite de la monarchie française. Cette source nous permet de saisir le processus de codification des mouvements de la personne du roi et de l'accessibilité aux espaces de sa vie quotidienne.

Dans l'article sur les Maronites, Guido Bellatti Ceccoli rend compte de la construction d'un espace culturel propre à une communauté de chrétiens arabophones arrivés à Livourne au début du XVIII ${ }^{\mathrm{e}}$ siècle. Les documents disponibles permettent de saisir la surprenante reconfiguration identitaire effectuée à Livourne par cette communauté migrante. Des épitaphes en arabe, particulièrement élaborées au niveau littéraire, témoignent d'un imaginaire spatial et émotionnel qui relie la Toscane et le Proche Orient, Alep et Livourne.

Ariane Devanthéry choisit d'analyser la représentation de l'espace lausannois dans les guides de voyage du XVIII e siècle à nos jours. Les auteurs de ces guides se trouvent partagés entre deux objectifs inconciliables, celui de raconter le voyage qu'ils ont eux-mêmes réalisé et celui de fournir un outil pragmatique pour des voyages à venir. Les 
postures auctoriales évoluent ainsi entre subjectivité et détachement descriptif, entre discours purement informatif et nécessité de hiérarchiser émotionnellement des points de repère.

L'étude de Katarzyna Matul, consacré à un objet d'art considéré comme mineur, l'affiche, compare les difficultés rencontrées en France et en Pologne pour consacrer à cet objet un espace d'exposition reconnu au niveau institutionnel. Les deux tentatives analysées montrent une interprétation très différente de l'espace muséal, gouverné par des tensions politiques, artistiques et commerciales souvent inconciliables.

Tiina Tuominiemi nous présente un cas de reconfiguration littéraire de l'espace représenté dans le Petit Poucet de Perrault. Dans le roman Sept frères, l'écrivain finlandais Kivi réoriente les connotations fantastiques de la forêt de Perrault pour lui permettre de faire référence à un espace géographique réel, typiquement finlandais. Cette opération trouve son sens à l'intérieur d'un discours motivé par la nécessité historique de donner une conscience nationale au peuple finlandais.

La linguiste Federica Diémoz étudie l'aire-carrefour des parlers franco-provençaux valaisans, où la tradition populaire du conte montre l'ancrage de la langue parlée au territoire. La pratique populaire du "contage" reproduit des récits déjà connus et les reconfigure dans un espace vécu et localement reconnaissable. L'analyse des toponymes, des préfixes et des adverbes déictiques montre une significative redondance de la référence spatiale où s'exprime l'identification communautaire au territoire.

Jenny Ponzo analyse la représentation littéraire de la place dans des romans portant sur la période de l'unification nationale italienne. Construite souvent comme un discours de propagande, avec ses monuments, son Hôtel de ville, son église, sa fontaine, ses routes d'accès et de sortie, la place est ici surtout un espace idéologique, marqué par des tensions politiques et sociales qui perdurent sous la façade d'un précaire équilibre institutionnel.

Silvia Calligaro nous parle de l'espace urbain contemporain représenté par deux poètes dialectaux du nord de l'Italie, Delio Tessa et Franco Loi. Sur la base d'un corpus choisi en fonction de la présence d'indicateurs spatiaux, on observe un espace industrialisé que le moi structure par sa propre flânerie jusqu'à le transformer en paysage allégorique.

L'article de Charlotte Bouchez sur la téléréalité en Suisse Romande étudie la création médiatique d'un espace d'identité régional comme 
expression d'une stratégie de résistance aux produits télévisés d'importation. Dans la téléréalité suisse romande des années 2000, la mise en valeur de l'espace régional répond à l'objectif idéologique et commercial de favoriser un processus d'auto-reconnaissance du public local. Dans une logique de protection téléculturelle, le public est le destinataire de clichés discursifs qui renvoient à l'espace idyllique d'une Suisse rassurante et aux valeurs traditionnelles du travail, de la famille et de la patrie.

La lecture de l'ensemble de ces essais nous montre un échantillon d'espaces dits «réels» qui se trouvent investis par des schémas de repérage culturel, par des besoins existentiels, par des usages sociaux et économiques qui les textualisent et les rendent lisibles. L'objectivation de ces espaces ne semble donc possible que si on les considère comme des systèmes complexes formés par plusieurs couches d'énonciations verbales et non verbales ou, autrement dit, comme des discours qu'on peut essayer de traduire ou de laisser partiellement émerger. Tous les cas particuliers étudiés dans la section "Représentations de l'espace» peuvent donc se relier à une même trame: le processus de création de marques spatiales et d'indicateurs d'orientation propre à toute expérience de reconnaissance identitaire, individuelle ou culturelle. C'est cette exigence de délimitation identitaire qui se matérialise en représentation spatiale. Pour cette raison, nous prenons finalement conscience que de la même manière qu'«il n'y a pas de hors-texte», selon le célèbre aphorisme derridien, il n'y a pas non plus de discours possible «hors-espace», ni, probablement, "hors-paysage».

Alberto Roncaccia

Université de Lausanne 
